\title{
Ceftriaxone Drug Utilization Evaluation (DUE) at Prince Abdulaziz Bin Moussae'ed Hospital, Arar, Kingdom of Saudi Arabia
}

\section{Muhammad Jan Shamsur Rehman ${ }^{1}$, Majed Alrowaili², Abdul Wajed Abdul Rauf $^{3}$, and Elhassan Hussein Eltom ${ }^{1}$}

${ }^{1}$ Department of Pharmacology, College of Medicine, Northern Border University, Arar, Kingdom of Saudi Arabia

${ }^{2}$ Department of Orthopedic, Prince Abdulaziz Bin Mossae'ed Hospital \& College of Medicine, Northern Border University, Arar, Kingdom of Saudi Arabia

${ }^{3}$ Internal Medicine, Prince Abdulaziz Bin Moussae'ed Hospital, Arar, KSA

Corresponding Author:

Muhammad Jan Shamsur Rehman;

Tel: 00966-0569100195

email: rmuhammad-

jansmc@gmail.com

Received 23 August 2019

Accepted 14 December 2019

Published 30 December 2019

Production and Hosting by

Knowledge E

(c) Muhammad Jan Shamsur Rehman et al. This article is distributed under the terms of the Creative Commons

Attribution License, which permits unrestricted use and redistribution provided that the original author and source are credited.

Editor-in-Chief:

Prof. Mohammad A. M. Ibnouf

\section{Abstract}

Introduction: Bacterial resistance to the most commonly used drug ceftriaxone and its adverse effects have increased due to its injudicial use. Drug Utilization Evaluation (DUE) is an ongoing, systematic process designed to promote the appropriate and effective use of drugs. The purpose was to detect potential problems and search out solution.

Methods: Data were collected in structured preform specific for DUE and were represented as numbers and percentages. For comparing numerical data, MannWhitney U-test was used as the data were non-parametric in distribution for ages and BMI, while Fisher exact test was used for categorical data comparison. Multivariate multi-logistic regression models to study the overlapped effect of the variables in development of Ceftriaxone induced liver functions derangement. Data were processed and analyzed using SPSS16 for statistical analysis. Significance was considered as $\mathrm{P}<0.05$.

Results: Our study showed that ceftriaxone use was more. High dose was used and for more than 10 days were the result of adverse effects and liver insult.

Conclusion There is need for awareness among the general practitioners and clinicians to ensure rational use of antibiotics. This will minimize bacterial resistance to drug, adverse effects and save national economy.

Keywords: Ceftriaxone, Drug utilization evaluation

\section{G OPEN ACCESS}

\section{Introduction}

Antibiotics are the most frequently prescribed drugs and more likely to be used inappropriately. The irrational use of antibiotics can lead to microbial resistance, patient morbidity and mortality, prolonged hospital stay, and wastage of resources. Out of many antibiotics, ceftriaxone, one of the third-generation cephalosporin, is a very commonly used and more commonly misused antibiotic. As per the WHO recommendations, Drug 
Utilization Evaluation (DUE) studies should be conducted for such commonly used drugs in order to detect their irrational use and suitable measures should be adopted for their safe prescription and to avoid any harmful sequels.

The DUE studies are meant to promote the appropriate and effective use of drugs. The main idea behind is to find out the possible problems in the prescription, administration of drugs and adopt strategies for their rational use. DUE studies are based on some qualitative measures for the detection of the problems in the use of drugs, as well as emphasize on the outcomes and cost-effectiveness of drug therapy.

The purpose is to ensure rational drug use. DUE contains qualitative measures and emphasizes the outcomes and cost-effectiveness of drug therapy [1]. DUE is performed in patient settings to identify trends of over use and in-appropriate prescribing. Surveys have shown that 22-65\% antibiotics prescriptions are either in-appropriate or incorrect. Taking this in account targeting the antibiotics that are prescribed frequently for DUE would be important to extend the useful life of the antimicrobial agents [2].

Limiting the emergence and spread of antibiotic resistance, managing medicines costs and improving medical care, all are the leading reasons to evaluate specific drug use. Cephalosporin are the most widely used antibiotics for treating common infections [3]. These are large group of related beta-lactam antimicrobial agents with broad spectrum of activity, low rates of toxicity and ease of administration [4]. During the past several years, the problem of antibiotic resistance has noticeably worsened in Korea [5]. Ceftriaxone is one of the most commonly used antibiotics Compared to infections caused by susceptible pathogens. Diseases caused by resistant pathogens are associated with higher rates of morbidity and mortality [6]. Inappropriate utilization of ceftriaxone, however, including continued empiric use for presumed infections and prophylactic perioperative injection remained high [7].

Data collection is done in structured preform based on criteria for DUE of the American Society of Health System Pharmacist [7] and WHO criteria for DUE [8].

In a study, Ceftriaxone was most frequently prescribed (64\%) third generation cephalosporin in parenteral form [9]. Third generation cephalosporin are the most commonly prescribed broad spectrum antibiotic even before the culture sensitivity results arrives [10]. DUE has been defined by the American Society of Health System Pharmacists [11]. DUE are conducted in patient settings to identify trends of over use, underuse, or inappropriate prescribing. Inappropriate use of antibiotics leads to the emergence of microbial resistance, patient's morbidity \& mortality, prolonged hospital stay and wastage of financial and other resources. Unscientific use of drugs may lead to many problems like side effects, toxic effects, drugs interactions \& occurrence of drugs 
induced diseases. Considering all these factors, it is of immense importance to conduct DUE studies on antibiotics, which are being prescribed quite frequently. Amongst the antibiotics, cephalosporin is the most widely used for the treatment of common infections. Cephalosporin comprises a large group of beta-lactam drugs ranging from first to fifth generation with broad spectrum of activity. Out of cephalosporin, ceftriaxone is one of the most commonly prescribed drugs for treatment of different types of bacterial infections. It is cephalosporin especially effective against gram negative bacteria. Inappropriate use of ceftriaxone is also common to occur and may include overuse, underuse, frequent empiric use for presumed infections and prophylactic perioperative injections. WHO has recommended that DUE studies must be done for the commonly used drugs to find out their possible irrational use and to take appropriate measures to prevent the undesirable outcomes. In the present study DUE of ceftriaxone was conducted at the Prince Abdulaziz Bin Moussa'ed Hospital, Arar, which is a tertiary care hospital in the Northern Border Region of Saudi Arabia, it was designed to perform DUE of ceftriaxone in order to get useful information about the irrational use of drug in the form of low dose, very high dose, use for very small duration or very long duration. It will to prevent the spread of microbial resistance, minimize adverse drug reactions. This will create more awareness among general practitioners, clinicians to ensure rational use of the drug \& save national economy.

\section{Materials and Methods}

A retrospective DUE of ceftriaxone was carried out in Prince Abdulaziz Bin Moussa'ed Hospital at Arar which is the capital of Northern Borders Province in Saudi Arabia that has a population of 145,237 . Data was collected in structured preform, based on the criteria for DUE of the American Society of Health System Pharmacist [7] and the WHO criteria for DUE [8]. Medical records of patients who received ceftriaxone during hospitalization between 1st January 2018 to $31^{\text {st }}$ December 2018, were scrutinized and the relevant information were entered in the prescribed preform. According to the standard criteria the justification of drug use, process indicators, and outcome measures were investigated along with patient's demographic data. Data was entered in excel file and represented as numbers and percent. For Comparison of numerical data Mann-Whitney $\mathrm{U}$ test was used as the data was non parametric in distribution for ages and BMI. While Fisher exact test was used for categorical data comparison. Multivariate multi-logistic regression models to study the overlapped effect of the variables in development of 
Ceftriaxone induced liver functions derangement. The data was processed and analyzed using SPSS16 for statistical analysis. Significance was considered with $\mathrm{P}<0.05$.

\section{Results}

DUE of ceftriaxone for the period of January $1^{\text {st }} 2018$ to December 31th 2018, 1141 patients admitted to prince Abdualaziz Bin Mussa'ed hospital in Arar city were prescribed ceftriaxone for different indications.

Table (1) represent the demographic data of those patients, 609 (53.4\%) male and 532 (46.6\%) female, the age group 20 - 39 years old constitute the majority of cases 421 (36.9 \%). Saudi patient were 989 (86.7\%) while non-Saudi were 152 (13.3 \%), allergy to medications was reported in 11 (1.1\%) patients.

TABLE 1: Demographic data of ceftriaxone encounters (total of 1141).

\begin{tabular}{|c|c|c|}
\hline Gender & No. & $\%$ \\
\hline M & 609 & 53.4 \\
\hline$F$ & 532 & 46.6 \\
\hline Age & No. & $\%$ \\
\hline$<20$ & 255 & 22.3 \\
\hline 20-39 & 421 & 36.9 \\
\hline $40-59$ & 219 & 19.2 \\
\hline $60-79$ & 187 & 16.4 \\
\hline$>80$ & 59 & 5.2 \\
\hline Nationality & No. & $\%$ \\
\hline Saudi & 989 & 86.7 \\
\hline Non-Saudi & 152 & 13.3 \\
\hline Allergies & No. & $\%$ \\
\hline $\begin{array}{l}\text { Allergies } \\
\text { - Penicillin (4) } \\
\text { - Ciprofloxacin (1) } \\
\text { - Metoclopramide (3) } \\
\text { - Ibuprofen (3) }\end{array}$ & 11 & 1.0 \\
\hline No allergies & 1130 & 99.0 \\
\hline
\end{tabular}

Table (2) shows the justification of ceftriaxone use, this drug had been used prophylactically in 106 (9.3\%), based on culture and sensitivity (C/S) in 13 (1.1\%), and prescribed empirically in 1022 (89.6 \%). This table also represents dose, duration, and route of administration of ceftriaxone, there were $18(1.6 \%)$ patient prescribed the drug for more 
than 14 days. The frequent dose was 2 grams/day in 1053 (92.3\%) while 41 (3.6 \%) had taken a dose of 4 grams/day.

TABLE 2: Justification of ceftriaxone use (total of 1141)

\begin{tabular}{l} 
Justification 4 \\
prophylactic \\
Culture: \\
- Bronchitis (3) \\
- Pneumonia (4) \\
- Tonsillitis (1) \\
- Chronic gastritis (1) \\
- Diabetic complication (2) \\
- Septic wound (2) \\
Empirical \\
Course duration \\
1 day \\
2 to 7 days \\
8 to 14 days \\
$>14$ days \\
Dosage (gram/day) \\
0.5 \\
1 \\
1.5 \\
2 \\
Route of administration \\
IVI \\
\hline
\end{tabular}

\begin{tabular}{|c|c|}
\hline No. & $\%$ \\
\hline 106 & 9.3 \\
\hline 13 & 1.1 \\
\hline 1022 & 89.6 \\
\hline No. & $\%$ \\
\hline 150 & 13.1 \\
\hline 780 & 68.4 \\
\hline 193 & 16.9 \\
\hline 18 & 1.6 \\
\hline No. & $\%$ \\
\hline 4 & 0.4 \\
\hline 35 & 3.1 \\
\hline 5 & 0.4 \\
\hline 1053 & 92.3 \\
\hline 3 & 0.3 \\
\hline 41 & 3.6 \\
\hline No. & $\%$ \\
\hline 886 & 77.7 \\
\hline 255 & 22.3 \\
\hline
\end{tabular}

IV: Intravenous, IVI: intravenous Infusion

In Table (3), process indicators of ceftriaxone use had been summarized, we observed that $\mathrm{C} / \mathrm{S}$ had been done only for 13 (1.1\%) patients, for the rest of the patient ceftriaxone either used empirically or prophylactically.

Table (4), shows trauma and fractures which were the highest and constitute 254 (22.3 $\%)$, followed by upper respiratory tract infection 154 (13.5\%), gastritis and gastroenteritis 132 (11.6\%), appendicitis 115 (10.1\%).

Table (5) shows the most common adverse drug reactions and safety profile of ceftriaxone in the studied cases, with remarks to liver function, renal function and complete blood count were extracted. Accordingly, 79 (6.9\%) of patients showed SGPT elevation, adjusted to those with normal SGPT on admission, 112 (9.8\%) of patients 
TABLE 3: Process indicators of ceftriaxone encounters (total of 1141).

Process indicator
Appropriate C\&S obtained within 48 hours
before initial ceftriaxone dose
Complete blood count (CBC) with differential
obtained before initial ceftriaxone dose
Renal function obtained if sever hepatic and
renal impairment occurs
Liver function obtained before initial
ceftriaxone dose
Vital signs monitored at least three time daily
until patient becomes afebrile and at least
once daily thereafter during ceftriaxone
therapy

\begin{tabular}{|c|c|}
\hline No. & $\%$ \\
\hline 13 & 1.1 \\
\hline 1141 & 100 \\
\hline 1141 & 100 \\
\hline 1141 & 100 \\
\hline 1141 & 100 \\
\hline
\end{tabular}

TABLE 4: Distribution of ceftriaxone encounters (total of 1141), according to diagnosis.

Diagnosis
Abscesses
Appendicitis
Biliary infections
COPD
Diabetic coma and diabetic foot
Gastritis \& gastroenteritis
Meningitis
Pneumonia
Trauma and fractures
Upper respiratory tract infections
UTI
Total

\begin{tabular}{|c|}
\hline Number \\
\hline 37 \\
\hline 115 \\
\hline 85 \\
\hline 92 \\
\hline 96 \\
\hline 132 \\
\hline 11 \\
\hline 63 \\
\hline 254 \\
\hline 154 \\
\hline 102 \\
\hline 1141
\end{tabular}

\begin{tabular}{|c|}
\hline$\%$ \\
\hline 3.2 \\
\hline 10.1 \\
\hline 7.4 \\
\hline 8.1 \\
\hline 8.4 \\
\hline 11.6 \\
\hline 1.0 \\
\hline 5.5 \\
\hline 22.3 \\
\hline 13.5 \\
\hline 8.9 \\
\hline 100
\end{tabular}

showed SGOT elevation, adjusted to those with normal SGOT on admission, 15 (0.12 \%) of patients show serum creatinine level elevation, adjusted to those with normal levels on admission, 9 (2.2 \%) showed thrombocytosis with exclusion of post-operative and trauma cases, and those treated with ceftriaxone for more than 8 days.

Table (6) represents the univariate and multivariate of our data, we found that age, body mass index (BMI), duration of treatment, and IV bolus route had significant correlation with ceftriaxone liver insult, with P. value of $0.0002,0.0008,0.0001$, and 0.0001 respectively. Figure (1) assure the same result using Man-Whitney $U$ test for age and BMI because the data were non parametric at those level, while fisher exact was used for the four rest categorical variables. For duration \& route, the $P$ value was very highly significant of all the parameters $(\mathrm{P}<0.0001)$. 
TABLE 5: Ceftriaxone safety profile on remarks to liver function, renal function and complete blood count.
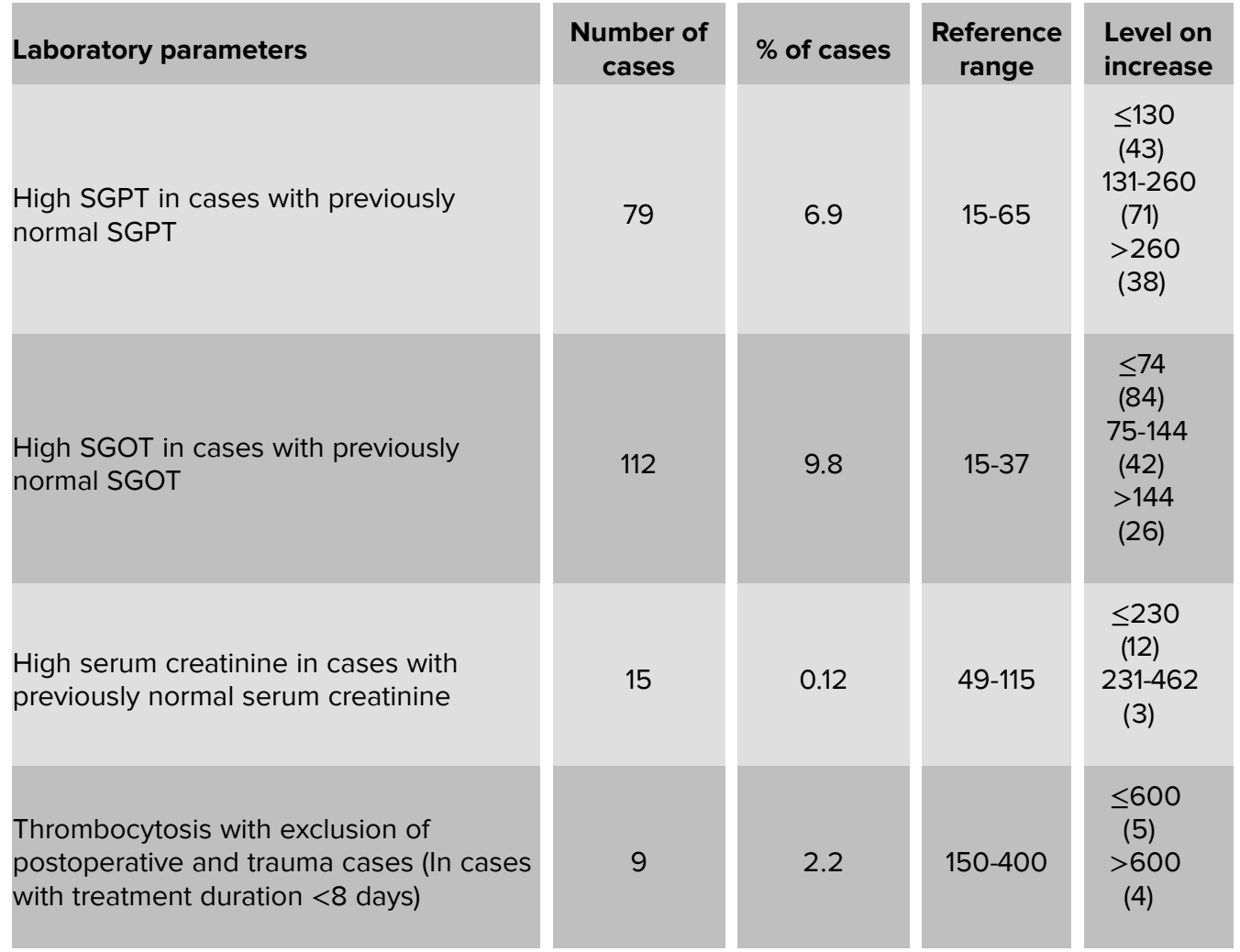

TABLE 6: Correlation of 6 independent variables with ceftriaxone liver insult using univariate and multivariate analysis.

Variables
Age
BMI
Dose(1)
Duration(1)
Gender(1)
Route(1)

\begin{tabular}{|c|}
\hline Univariate \\
\hline 0.0002 \\
\hline 0.0008 \\
\hline 0.5 \\
\hline$<0.0001$ \\
\hline 0.662 \\
\hline$<0.0001$
\end{tabular}

\begin{tabular}{|c|c|}
\hline \multicolumn{2}{|c|}{ Multivariate } \\
\hline Sig. & OR \\
\hline .021 & 1.617 \\
\hline .003 & 1.205 \\
\hline .030 & 1.372 \\
\hline .010 & 2.123 \\
\hline .791 & 0.210 \\
\hline .121 & 0.417 \\
\hline
\end{tabular}

\section{Discussion}

Drug use evaluation (DUE) is an on-going, authorized and systematic quality improvement process, which is designed to review drug use and/or prescribing patterns, provide feedback of results to clinicians and other relevant groups, develop criteria and standards which describe optimal drug use and promote appropriate drug use through education and other interventions. Cephalosporin is the most commonly used antibiotics. In a study in Arab Emirate, it was observed that 3rd generation cephalosporin 
constituted $83.6 \%$ of the prescriptions, with ceftriaxone being the most commonly used one (81.1\%). In another study it was $87 \%$. They were mainly prescribed for the treatment of the lower respiratory tract infections (60.2\%). Seven (3.5\%) different ADRs linked to cephalosporin use were observed ranging from oral thrush to clostridium difficile infection. The $3^{\text {rd }}$ generation cephalosporin was commonly prescribed by parenteral route [12]. Our study is in complete agreement with the above study that states that ceftriaxone is the most commonly used drug in Saudis (86.7\%) \& in above study it was 81.1\%. The percentage is more for Saudi people because as compared to non-Saudis, most of the patients coming to the government hospital are Saudis. Similarly, in above study adverse drug reactions to ceftriaxone were $3.5 \%$ but in our study they were $1.1 \%$. Likewise, the most common route of administration was parenteral in our study which is in agreement with the above mentioned study. Demographic details of a study for DUE of ceftriaxone shows that males were $54.5 \%$ and females were $45.5 \%$ [13]. Our study is in complete agreement with above study which shows male $53.4 \%$ \& female 46.6\%. In our study we found that dose \& duration of ceftriaxone were $1.6 \%$ in patients prescribed the drug for more than 14 days. The frequent dose was 2 grams/day in $92.3 \%$ while $3.6 \%$ had taken a dose of 4 grams/day. This overdose \& long duration may lead to increased side effects \& wastage of drug. In a cross sectional study, it was found out that in $56 \%$ patients, ceftriaxone was prescribed without indication [14]. Antimicrobial drug resistance has been projected to add between $\$ 100$ million and $\$ 30$ billion annually to health-care costs [15]. In a study it was observed that use of the drug was $89 \%$ in age $15-65$ years \& $2.3 \%$ in patients above 65 years [14]. Our study is consistent with this study in which we found the same as $36.9 \%$ for age $20-39$ years \& $5.2 \%$ in patients above 80 years. Our study showed most common adverse drug reactions and safety profile of ceftriaxone with remarks to liver function, renal function and complete blood count. The patients showed $6.9 \%$ ) SGPT elevation, $9.8 \%$ of patients showed SGOT elevation, $0.12 \%$ ) of patients showed serum creatinine level elevation, $2.2 \%$ ) showed thrombocytosis all treated with ceftriaxone for more than 8 days. So, these side effects will be controlled if the drug is not used for unnecessary long duration.

Similarly, in our study we observed that C/S had been done only for $1.1 \%$ patients, for the rest of the patients ceftriaxone either used empirically or prophylactically. So, culture \& sensitivity if done for all the patients will ensure required \& judicial use of drug. In our study trauma and fractures were the highest and constitute $22.3 \%$, for which ceftriaxone was prescribed. Similarly, in our study we found that age, body mass index (BMI), duration of treatment, and IV bolus route had significant correlation with ceftriaxone liver insult, with P. value of $0.0002,0.0008,0.0001$, and 0.0001 respectively. For duration \& route, 
the $P$ value was very highly significant of all the parameters $(P<0.0001)$. In many other studies it is clear that high dose \& duration of treatment for more than 10 days will lead to more side effects.

\section{Conclusion}

Our study emphasized the need for creating more awareness among the general practitioners and clinicians to ensure rational use of antibiotics to minimize resistance, adverse drugs reactions \& save national economy. Therefore, we recommend that a team, particularly the clinical pharmacists in all wards should monitor \& ensure the judicial use of ceftriaxone and other antibiotics.

\section{Acknowledgement}

This project was funded by deanship of Scientific Research, Northern Border University for their financial support under grant No. (6984-med-2017-1-7-F). The authors, therefore, acknowledge with thanks DSR technical and financial support.

\section{Authors' Contribution}

Author nos. 1-3 handled the design, data collection, interpretation, arranging \& rechecking.

Author no.4 handled the statistical analysis and typing

\section{Grant \& Support}

The study has been funded by the Deanship of Research, Northern Border University, Arar, Kingdom of Saudi Arabia.

\section{References}

[1] Qingping, S., Feng, D., Ran, S., et al. (2013). Drug use evaluation of cefepime in the first affiliated hospital of Bengbu medical college: A retrospective and prospective analysis. BMC Infectious Diseases, vol. 13, no. 1, p. 160

[2] Lee, H., Jung, D., Yeom, J. S., et al. (2009). Evaluation of ceftriaxone utilization at multi-center study. Korean Journal of Internal Medicine, vol. 24, no. 4, pp. 374-380. 
DOI: 2009;10, 3904.Kjim

[3] Laxminarayan, R., Bhutta, Z., Duse, A., et al. (2005). Drug resistance, in Disease Control Priorities in Developing Countries, pp. 1031-1051.

[4] William, F. and Janis, E. (1996). The cephalosporins. Mayo Clinic Proceedings, vol. 74, pp. 187-195.

[5] Chong, Y. and Lee, K. (2000). Present situation of antimicrobial resistance in Korea. Journal of Infection and Chemotherapy, vol. 6, pp. 189-195.

[6] Holmberg, S. D., Solomon, S. L., and Blake, P. A. (1987). Health and economic impacts of antimicrobial resistance. Reviews of Infectious Diseases, vol. 9, pp. 1065-1078.

[7] American Society of Health-System Pharmacists. (1996). ASHP guidelines on medication-use evaluation. American Journal of Health-System Pharmacy, vol. 53, pp. 1953-1955.

[8] Holloway, K. and Green, T. (2003). Drug and Therapeutics Committees - A Practical Guide. Geneva, Switzerland: World Health Organization, Department of Essential Drugs and Medicines Policy.

[9] Panavila, L., Ninan, N., and Pratima, K. C. (2016). Drug use evaluation of third generation cephalosporin in a tertiary care teaching hospital . International Journal of Therapeutic Applications, vol. 32, pp. 81-85.

[10] Kaliamoorthy, K., Sankaralingam, R., Punniyakotti, S., et al. (2012). Drug utilization evaluation of third generation cephalosporins using core drug use indicators. Pakistan Journal of Pharmaceutical Sciences, vol. 25, no. 2, pp. 339-342.

[11] Amos, M. Y., Yitzhack, S., Yechiel, S., et al. (2000). Cefuroxime utilization evaluation: Impact of physician Education on prescribing patterns. IMAJ, vol. 2, pp. 187-191.

[12] Abou-Shaaban, M., Ali, A. A., Rao, P. G., et al. (2016). Drug utilization review of cephalosporins in a secondary care hospital in United Arab Emirates. International Journal of Clinical Pharmacy, vol. 38, no. 6, pp. 1367-1371.

[13] Naveen, V., Abubakar, S., and Chandana, G. (2018). Drug utilization pattern of cephalosporin. International Journal of Current Pharmaceutical Research, vol. 10, no. 3, pp. 33-36.

[14] Hafte, K., Tefera, K., Azeb, W., et al. (2018). Assessment of ceftriaxone use in Eastern Ethiopian Referral Hospital: A retrospective study. Journal of Infectious Diseases and Medical Microbiology, vol. 2, no. 3, pp. 26-29.

[15] Phelps, C. E. (1989). Bug/drug resistance: Sometimes less is more. Medical Care, vol. 27, pp. 194-203. 